\title{
INSERTION, APPROXIMATION, AND EXTENSION OF REAL-VALUED FUNCTIONS
}

\author{
ROBERT L. BLAIR AND MARY ANNE SWARDSON
}

\begin{abstract}
For a uniformly closed vector lattice $V$ of real-valued functions on a set $X$, necessary and sufficient conditions are obtained for insertion (or "strict insertion") of some member of $V$ between two arbitrary real-valued functions on $X$. These conditions quickly yield known insertion, approximation, and extension theorems for real-valued functions.
\end{abstract}

1. Introduction. For $X$ a set, $F(X)$ (resp. $\left.F^{*}(X)\right)$ denotes the set of all realvalued (resp. bounded real-valued) functions on $X$. We view $F(X)$ as both a vector lattice and ring (with the pointwise operations) and as a topological space with the topology of uniform convergence. A "vector sublattice" or "subring" of $F(X)$ will always be understood to contain all the constant real-valued functions on $X$.

For $f \in F(X)$ and $a \in \mathbf{R}$, set $L_{a}(f)=\{x \in X: f(x) \leq a\}, L^{a}(f)=\{x \in$ $X: f(x) \geq a\}$, and $Z(f)=\{x \in X: f(x)=0\}$. Sets of the form $L_{a}(f)$ (resp. $\left.L^{a}(f)\right)$ are lower (resp. upper) Lebesgue sets of $f$.

For $V$ a vector sublattice of $F(X)$, uc $V$ denotes the uniform closure of $V$ in $F(X)$ and (following [Ma, p. 51]) us $V$ (resp. ls $V$ ) denotes the set of all $f \in F(X)$ such that $f$ is the pointwise limit of some decreasing (resp. increasing) sequence of functions in $V$. We say that $V$ is inversion-closed (resp. closed under (bounded) quotients) if $1 / g \in V$ whenever $g \in V$ with $Z(g)=\varnothing$ (resp. $f / g \in V$ whenever $f, g \in V$ (with $f / g$ bounded) and $Z(g)=\varnothing$ ). If $f, g \in F(X)$, then $V$ completely separates the Lebesgue sets of the pair $\langle f, g\rangle$ if for every $a<b$ in $\mathbf{R}, L_{a}(g)$ and $L^{b}(f)$ are completely separated by some function in $V$ (i.e. there exists $k \in V$ such that $k=0$ on one of the sets $L_{a}(g)$ and $L^{b}(f), k=1$ on the other, and $\left.0 \leq k \leq 1\right)$, and $V$ completely separates the Lebesgue sets of $f$ if $V$ completely separates the Lebesgue sets of $\langle f, f\rangle$. By a $V$-zero-set we mean a set of the form $Z(f)$ with $f \in V$.

For a topological space $X$ and $S \subset X$, set $C(X)=\{f \in F(X): f$ is continuous $\}$, $C^{*}(X)=C(X) \cap F^{*}(X), C(X) \mid S=\{f \mid S: f \in C(X)\}$, and $C^{*}(X) \mid S=\{f \mid S: f \in$ $\left.C^{*}(X)\right\}$. As usual, $S$ is $C$-embedded (resp. $C^{*}$-embedded) in $X$ if $C(S)=C(X) \mid S$ (resp. $\left.C^{*}(S)=C^{*}(X) \mid S\right)$. By a zero-set of $X$ we mean a $C(X)$-zero-set.

We begin by quoting two basic results:

1.1. APPROXIMATION THEOREM. Let $X$ be a set, let $V$ be a vector sublattice of $F(X)$, and let $f \in F(X)$. If $V$ completely separates the Lebesgue sets of $f$, and if either $f \in F^{*}(X)$ or uc $V$ is inversion-closed, then $f \in$ uc $V$.

Received by the editors November 21, 1983 and, in revised form, February 2, 1984. Presented at the Spring Topology Conference, Auburn University, March 22, 1984.

1980 Mathematics Subject Classification. Primary 54C30; Secondary 46A40, 54C05, 54C08, 54C20.

Key words and phrases. Lebesgue sets, vector lattices of functions, rings of functions, uniform convergence, approximation, extension, insertion, strict insertion, zero-sets.

(C)1985 American Mathematical Society $0002-9939 / 85 \$ 1.00+\$ .25$ per page 
1.2. TOpological Insertion TheOREm. If $X$ is a topological space and if $f, g \in F(X)$, then the following are equivalent:

(1) There exists $h \in C(X)$ such that $f \leq h \leq g$.

(2) For every $a<b$ in $\mathbf{R}, L_{a}(g)$ and $L^{b}(f)$ are completely separated in $X$.

1.3. REMARKs. (a) 1.1 is due to Mrówka (for $f \in F^{*}(X)$ see $[\mathbf{M}, 2.7]$ and for uc $V$ inversion-closed see $[\mathbf{M}, 3.3]$ and 2.2 below). Consequences of 1.1 include the Extension Theorem (1.4 below) as well as several versions of the Stone-Weierstrass Theorem (see e.g. [M, 4.1, 4.6, and 4.8]).

(b) For an example of an inversion-closed vector lattice of functions whose uniform closure is not inversion-closed, see 3.6 below.

(c) 1.2 is due independently to Blair $[\mathbf{B l}, 3.5]$ and Lane $\left[\mathbf{L}_{1}, 2.1\right]$. (Its proofs in $[\mathbf{B l}]$ and $\left[\mathbf{L}_{1}\right]$ are based on techniques of Tong $[\mathbf{T}]$ and Katětov $\left[\mathbf{K}_{\mathbf{1}}\right]$ (correction $\left[\mathbf{K}_{2}\right]$ ), respectively.) 1.2 generalizes earlier results of Katětov $\left[\mathbf{K}_{1}, \mathbf{K}_{2}\right]$ and Tong $[\mathbf{T}$, Theorem 2] for normal spaces (cf. Engelking [E, 1.7.15(b)] and Bourbaki [Bo, Chapter IX, §4, Exercise 30]), Dieudonné [D, Theorem 9] for paracompact spaces, and Hahn [H, p. 103] for metric spaces.

We next record a basic extension theorem. It is due to Mrówka $[\mathbf{M}, 4.11]$ and obviously implies the Gillman-Jerison version of Urysohn's Extension Theorem [GJ, 1.17] (namely, that a subset $S$ of a space $X$ is $C^{*}$-embedded in $X$ if and only if any two completely separated subsets of $S$ are completely separated in $X$ ).

1.4. EXTENSION THEOREM. If $X$ is a topological space, $S \subset X$, and $f \in$ $C^{*}(S)$, then the following are equivalent:

(1) $f$ has a continuous extension over $X$.

(2) For every $a<b$ in $\mathbf{R}, L_{a}(f)$ and $L^{b}(f)$ are completely separated in $X$.

We note that the nontrivial implication $(2) \Rightarrow(1)$ of 1.4 is a consequence of either 1.1 or 1.2 . Its easy proof from 1.2 is given in [Bl, p. 67], and its proof from 1.1 (as in $[\mathbf{M}])$ is immediate in view of $1.5(1)$ :

1.5. Proposition. Let $X$ be a topological space and let $S \subset X$.

(1) $C(X) \mid S$ is uniformly closed in $F(S)[\mathbf{M}, 4.10]$.

(2) $C(X) \mid S$ is inversion-closed if and only if $S$ is completely separated from every zero-set of $X$ disjoint from $S[\mathbf{M}, 4.15]$.

For a self-contained proof of 1.4 , see [Bl, 3.2]. We note also that, in view of 1.5, 1.1 quickly yields the nontrivial half of the Gillman-Jerison characterization of $C$-embedding [GJ, 1.18] (namely, that a subset $S$ of a space $X$ is $C$-embedded in $X$ if and only if $S$ is $C^{*}$-embedded in $X$ and completely separated from every zero-set of $X$ disjoint from $S$ ).

To summarize, we have the following diagram:

Approximation Theorem

Topological Insertion Theorem

Extension Theorem

The principal object of this paper is to complete the foregoing diagram with the following topology-free insertion theorem, which quickly yields both the Approximation Theorem and the Topological Insertion Theorem. 
1.6. INSERTION THEOREM. Let $X$ be a set, let $V$ be a uniformly closed vector sublattice of $F(X)$, and let $f, g \in F(X)$. If either $f, g \in F^{*}(X)$ or $V$ is inversionclosed, then the following are equivalent:

(1) There exists $h \in V$ such that $f \leq h \leq g$.

(2) $V$ completely separates the Lebesgue sets of $\langle f, g\rangle$.

We prove 1.6 in $\S 2$. Note that if $V$ is a vector sublattice of $F(X)$, then so is uc $V$, and hence 1.1 follows immediately from 1.6. Moreover, since $C(X)$ is a uniformly closed inversion-closed vector sublattice of $F(X), 1.2$ is also an immediate consequence of 1.6 .

Some results on "strict insertion" (which are related to 1.6 and which generalize results of Lane $\left.\left[\mathbf{L}_{2}\right]\right)$ are obtained in $\S 3$.

2. Proof of the Insertion Theorem. We first prove three lemmas.

2.1. LEMMA. If $V$ is a vector sublattice of $F(X)$, if $f, g \in F^{*}(X)$, and if $V$ completely separates the Lebesgue sets of $\langle f, g\rangle$, then there exists a decreasing sequence $\left\langle f_{n}: n \in \omega\right\rangle$ of functions in $V$ such that:

(1) For every $n \in \omega, f_{n}-g \leq 2^{-n}$.

(2) $f \leq \bigwedge_{n \in \omega} f_{n} \leq g$.

(3) $V$ completely separates the Lebesgue sets of $\left\langle\bigwedge_{n \in \omega} f_{n}, g\right\rangle$.

PROOF. It is easy to see that $f \leq g$, and thus there exist integers $p$ and $q$ such that $p \leq f \leq g \leq q$. For each $n \in \omega$ and each $m \in \omega$ with $m<(q-p) 2^{n+1}$, the hypotheses on $V$ imply that there exists $f_{m n} \in V$ such that

$$
\begin{aligned}
& f_{m n}=p+(m+1) 2^{-n-1} \quad \text { on }\left\{x \in X: g(x) \leq p+m 2^{-n-1}\right\}, \\
& f_{m n}=q \quad \text { on }\left\{x \in X: f(x) \geq p+(m+1) 2^{-n-1}\right\},
\end{aligned}
$$

and

$$
p+(m+1) 2^{-n-1} \leq f_{m n} \leq q .
$$

For each $n \in \omega$, let $f_{n}^{*}=\bigwedge\left\{f_{m n}: m<(q-p) 2^{n+1}\right\}$, let $f_{n}=\bigwedge_{i \leq n} f_{i}^{*}$, and note that $\left\langle f_{n}: n \in \omega\right\rangle$ is a decreasing sequence of functions in $V$ such that $f \leq f_{n}$ for every $n \in \omega$. Thus $f \leq \bigwedge_{n \in \omega} f_{n}$.

To verify (1), let $n \in \omega$ and $x \in X$. Then $f_{n}^{*}(x)=f_{m n}(x)$ for some $m<$ $(q-p) 2^{n+1}$. Now either $g(x)>q-2^{-n-1}$ or $p+k 2^{-n-1} \leq g(x) \leq p+(k+1) 2^{-n-1}$ for some $k \in \omega$ with $k+1<(q-p) 2^{n+1}$. In the first case,

$$
f_{n}(x)-g(x) \leq f_{m n}(x)-g(x)<q-q+2^{-n-1}<2^{-n},
$$

and in the second,

$$
f_{n}(x)-g(x) \leq f_{k+1, n}(x)-g(x) \leq p+(k+2) 2^{-n-1}-\left(p+k 2^{-n-1}\right)=2^{-n} .
$$

Thus (1) holds, and from (1) we conclude that $\bigwedge_{n \in \omega} f_{n} \leq g$.

Finally, to verify (3), let $a, b \in \mathbf{R}$ with $a<b$ (and we may assume that $p \leq a<$ $b \leq q)$. Pick $k \in \omega$ with $2^{-k}<b-a$ and let

$$
m=\max \left\{n \in \omega: p+n 2^{-k-1} \leq(a+b) / 2\right\} .
$$

Then $a<p+m 2^{-k-1}<p+(m+1) 2^{-k-1}<b, L_{a}(g) \subset\left\{x \in X: f_{m k}(x)=p+\right.$ $\left.(m+1) 2^{-k-1}\right\} \subset\left\{x \in X: f_{k}(x) \leq p+(m+1) 2^{-k-1}\right\}$, and $L^{b}\left(\bigwedge_{n \in \omega} f_{n}\right) \subset L^{b}\left(f_{k}\right)$. But since $f_{k} \in V$, it is clear that $V$ completely separates the Lebesgue sets of $f_{k}$, 
and thus $L_{a}(g)$ and $L^{b}\left(\bigwedge_{n \in \omega} f_{n}\right)$ are completely separated by some function in $V$.

For the proof of the following lemma, see e.g. [Ha, 2.4 and $\mathbf{M}$, Remark 2 and $3.2]$.

2.2. LEMMA. If $V$ is a uniformly closed subset of $F(X)$ and if either $V \subset$ $F^{*}(X)$ or $V$ is inversion-closed, then $V$ is a vector lattice if and only if $V$ is a ring.

We define the map $\gamma: \mathbf{R} \rightarrow(-1,1)$ by the formula $\gamma(t)=t /(1+|t|)$ for $t \in \mathbf{R}$. Clearly $\gamma$ is an order-preserving homeomorphism from $\mathbf{R}$ onto the open interval $(-1,1)$, and its inverse is given by the formula $\gamma^{-1}(s)=s /(1-|s|)$ for $s \in(-1,1)$. By 2.2 we have

2.3. LEMMA. Let $V$ be a uniformly closed inversion-closed vector sublattice of $F(X)$. Then $V$ is closed under quotients, and if $h \in V$ with $h(X) \subset(-1,1)$, then $\gamma^{-1} \circ h \in V$.

PROOF OF 1.6. (1) $\Rightarrow(2)$. Let $h$ be as in (1) of 1.6. Since $V$ completely separates the Lebesgue sets of $h,(2)$ follows from the inequalities $f \leq h \leq g$.

$(2) \Rightarrow(1)$. We first consider the case in which $f, g \in F^{*}(X)$. By (2) and 2.1, there exists a decreasing sequence $\left\langle f_{n}: n \in \omega\right\rangle$ of functions in $V$ that satisfies (2) and (3) of 2.1. Then clearly $V$ completely separates the Lebesgue sets of $\left\langle-g,-\bigwedge_{n \in \omega} f_{n}\right\rangle$, so by 2.1 again there is a decreasing sequence $\left\langle g_{n}^{\prime}: n \in \omega\right\rangle$ of functions in $V$ such that $g_{m}^{\prime}+\bigwedge_{n \in \omega} f_{n} \leq 2^{-m}$ for every $m \in \omega$ and $-g \leq \bigwedge_{n \in \omega} g_{n}^{\prime} \leq-\bigwedge_{n \in \omega} f_{n}$. Set $g_{n}=-g_{n}^{\prime}$. Then $\left\langle g_{n}: n \in \omega\right\rangle$ is an increasing sequence of functions in $V$ such that $f \leq \bigwedge_{n \in \omega} f_{n} \leq \bigvee_{n \in \omega} g_{n} \leq g$ and

$$
\bigwedge_{n \in \omega} f_{n} \leq g_{m}+2^{-m} \text { for every } m \in \omega .
$$

Next, there exists (recursively) a sequence $\left\langle h_{n}: n \in \omega\right\rangle$ of functions in $V$ such that $h_{0}=f_{0} \wedge g_{0}$ and such that, for every $n>0$,

$$
h_{n}=\left(\bigvee_{i \leq n}\left(f_{i} \wedge g_{i}\right)\right) \wedge\left(h_{n-1}+2^{-n}\right)
$$

Note that for every $n \in \omega, h_{n} \leq \bigvee_{i \leq n} g_{i} \leq g$ and that (inductively) $h_{n} \leq h_{n+1}$. Set $h=\bigvee_{n \in \omega} h_{n}$ and observe that $h \leq g$.

We claim that $h \in V$. For this it suffices to show that the (increasing) sequence $\left\langle h_{n}: n \in \omega\right\rangle$ converges uniformly to $h$; and hence it suffices to show that $\left\langle h_{n}: n \in \omega\right\rangle$ is uniformly Cauchy: For $\varepsilon>0$, pick $m \in \omega$ with $\sum_{i=m+1}^{\infty} 2^{-i}<\varepsilon$ and note that if $m \leq j<k$, then

$$
\left|h_{k}-h_{j}\right| \leq \sum_{i=j}^{k-1}\left|h_{i+1}-h_{i}\right| \leq \sum_{i=j}^{k-1} 2^{-i-1}<\varepsilon .
$$

It remains only to show that $f \leq h$. Let $x \in X$. If $h_{k}(x) \geq f_{k}(x)$ for some $k \in \omega$, then $f(x) \leq f_{k}(x) \leq h(x)$, so we may assume that $h_{k}(x)<f_{k}(x)$ for every $k \in \omega$. Moreover, if for every $n \in \omega$ there exists $k \geq n$ such that $h_{k}(x)=g_{k}(x)$, then $f(x) \leq \bigvee_{n \in \omega} g_{n}(x)=\bigvee_{n \in \omega} h_{n}(x)=h(x)$, so we may also assume that there exists a (least) $m \in \omega$ such that $h_{k}(x) \neq g_{k}(x)$ for every $k \geq m$. Clearly $m>0$ 
and, by induction, $h_{n}(x)=g_{m-1}(x)+\sum_{i=m}^{n} 2^{-i}$ for every $n \geq m$. Then by $(*)$ we have

$$
\begin{aligned}
f(x) & \leq \bigwedge_{n \in \omega} f_{n}(x) \leq g_{m-1}(x)+2^{-m+1} \\
& =g_{m-1}(x)+\sum_{i=m}^{\infty} 2^{-i}=\bigvee_{n \geq m} h_{n}(x)=h(x),
\end{aligned}
$$

and we conclude that $f \leq h$.

Now assume that $V$ is inversion-closed and that (merely) $f, g \in F(X)$. Since $\gamma \circ f, \gamma \circ g \in F^{*}(X)$ and $V$ completely separates the Lebesgue sets of $\langle\gamma \circ f, \gamma \circ g\rangle$, by what was just proved there exists $h \in V$ such that $\gamma \circ f \leq h \leq \gamma \circ g$. Then $\gamma^{-1} \circ h \in V$ by 2.3 , and $f \leq \gamma^{-1} \circ h \leq g$.

2.4. REMARKS. Parts of the preceding proof were suggested by the Tong-Blair proof of 1.2 (see 1.3(c)). But Tong's technique (in the proof of [ $\mathbf{T}$, Theorem 2]) is ultimately inadequate for a proof of $(2) \Rightarrow(1)$ of 1.6 since it merely produces an $h \in$ us $V \cap$ ls $V$ with $f \leq h \leq g$, and such an $h$ need not be in $V$, as the following example shows: Let $X=(0,1]$, define $h \in F(X)$ by $h(x)=\sin (1 / x)$, and let $V=C(\mathbf{R}) \mid X$. Then $V$ is a uniformly closed vector sublattice of $F(X)$ (by $1.5(1)$ ), $h \in$ us $V \cap \operatorname{ls} V$ (as is easily seen), but $h \notin V$. (The Katětov-Lane proof of 1.2 (see 1.3(c)) also does not appear to be readily adaptable to a proof of 1.6.)

3. Strict insertion. For $f, g, h \in F(X), h$ is strictly between $f$ and $g$ if $f \leq$ $h \leq g$ and $f(x)<h(x)<g(x)$ whenever $x \in X$ with $f(x)<g(x)$.

Our first result on "strict insertion" is an easy corollary of 1.6:

3.1. THEOREM. Let $V$ be a uniformly closed vector sublattice of $F(X)$ and let $f, g \in F(X)$. If either $f, g \in F^{*}(X)$ or $V$ is inversion-closed, then the following are equivalent:

(1) There exists $h \in V$ such that $h$ is strictly between $f$ and $g$.

(2) There exists $k \in F(X)$ such that $k$ is strictly between $f$ and $g$ and such that $V$ completely separates the Lebesgue sets of both $\langle f, k\rangle$ and $\langle k, g\rangle$.

PROOF. (1) $\Rightarrow(2)$. It suffices to take $k=h$.

$(2) \Rightarrow(1)$. By (2) and 1.6 there exist $h_{1}, h_{2} \in V$ such that $f \leq h_{1} \leq k \leq h_{2} \leq g$. Then $h=\left(h_{1}+h_{2}\right) / 2$ is in $V$ and $h$ is strictly between $f$ and $g$.

In what follows we generalize techniques and results of Lane $\left[\mathbf{L}_{2}\right]$.

The next lemma is for the most part implicit in the proof of Lane $\left[\mathbf{L}_{2}\right.$, Theorem 2], and in the bounded case is also a consequence of Mauldin [Ma, Theorem 4] (cf. Hager [Ha, p. 762]). It generalizes earlier results of Tong for perfectly normal spaces [T, Theorem 3], Hahn for metric spaces [H, p. 100], and Baire for the real line [Ba, p. 125]. We give a brief proof based on the proof of [T, Theorem 3].

3.2. LEMMA. Let $V$ be a uniformly closed vector sublattice of $F(X)$ and let $f \in F(X)$. If either $f \in F^{*}(X)$ or $V$ is inversion-closed, then the following are equivalent:

(1) Every upper (resp. lower) Lebesgue set of $f$ is a $V$-zero-set.

(2) $f \in$ us $V$ (resp. $f \in \operatorname{ls} V$ ).

PROOF. (1) $\Rightarrow(2)$. If $f \in F^{*}(X)$, then there exists $r \in \mathbf{R}$ with $r>0$ and $f(X) \subset(-r, r)$. Define (the order-preserving homeomorphism) $\tau:(-r, r) \rightarrow(0,1)$ 
by the formula $\tau(t)=(r+t) / 2 r$ for $t \in(-r, r)$. By (1) and the proof of $(1) \Rightarrow(2)$ of [T, Theorem 3], there exists a decreasing (resp. increasing) sequence $\left\langle f_{n}: n \in \omega\right\rangle$ of functions in $V$ whose pointwise limit is $\tau \circ f$ and with $f_{n}(X) \subset(0,1)$ for every $n \in \omega$. Then $\left\langle\tau^{-1} \circ f_{n}: n \in \omega\right\rangle$ is a decreasing (resp. increasing) sequence of functions in $V$ whose pointwise limit is $f$.

If, on the other hand, $V$ is inversion-closed, then by 2.3 and what was just proved (with $r=1$ ), $\left\langle\gamma^{-1} \circ \tau^{-1} \circ f_{n}: n \in \omega\right\rangle$ is again a decreasing (resp. increasing) sequence of functions in $V$ whose pointwise limit is $f$.

$(2) \Rightarrow(1)$. Assume there is a decreasing sequence $\left\langle f_{n}: n \in \omega\right\rangle$ of functions in $V$ with pointwise limit $f$. Let $a \in \mathbf{R}$, for each $n \in \omega$ set $g_{n}=1 \wedge\left(a-\left(f_{n} \wedge a\right)\right)$, and let $g=\sum_{n \in \omega} 2^{-n} g_{n}$. Then $g \in V$ and $L^{a}(f)=\bigcap_{n \in \omega} Z\left(g_{n}\right)=Z(g)$. The other case is proved similarly.

3.3. REMARKS. (a) The equivalence (ii) $\Leftrightarrow$ (iii) of $\left[\mathbf{L}_{2}\right.$, Theorem 2] is essentially the special case of 3.2 for which $X$ is a topological space, $f \in F^{*}(X)$, and $V=$ $C^{*}(X)$.

(b) For $f \in F^{*}(X),(1) \Rightarrow(2)$ of 3.2 holds even if $V$ is not uniformly closed (as can be seen from the proof of $[\mathbf{T}$, Theorem 3]); and in fact (1) $\Rightarrow(2)$ holds if $f$ is merely bounded above (resp. below) (see [Ma, Theorem 4]).

The following lemma is clear.

3.4. LEMMA. If $V$ is a vector sublattice of $F(X)$ that is closed under bounded quotients and if $u, v \in V$ with $Z(u) \cap Z(v)=\varnothing$, then the function $|u| /(|u|+|v|)$ is in $V$ and completely separates $Z(u)$ and $Z(v)$.

3.5. COROLlARY. If $V$ is a vector sublattice of $F(X)$ and if $V$ is closed under bounded quotients (resp. if uc $V$ is inversion-closed), then us $V \cap \operatorname{ls} V \cap F^{*}(X) \subset$ uc $V$ (resp. us $V \cap \operatorname{ls} V \subset$ uc $V)$.

PROOF. This is immediate from 3.2, 3.4, 1.1, and 2.3.

3.6. EXAMPLE. We give an example of a set $X$ and an inversion-closed vector sublattice $V$ of $F(X)$ such that us $V \cap \operatorname{ls} V \not \subset$ uc $V$ (and hence with uc $V$ not inversion-closed by 3.5): Let $X$ and $h$ be as in 2.4. Clearly there exist increasing and decreasing sequences $\left\langle f_{n}: n \in \omega\right\rangle$ and $\left\langle g_{n}: n \in \omega\right\rangle$ of functions in $C(X)$, both pointwise convergent to $h$, such that each $f_{n}$ (resp. $g_{n}$ ) is -1 (resp. 1) on some neighborhood of 0 . Let $W$ be the smallest lattice-ordered subring of $F(X)$ that contains $\left\{f_{n}: n \in \omega\right\} \cup\left\{g_{n}: n \in \omega\right\}$ and let $V=\{p / q: p, q \in W$ and $Z(q)=\varnothing\}$. One can verify that $V$ is an inversion-closed vector sublattice of $F(X)$ and that each function in $V$ is constant on some neighborhood of 0 . Hence $V \subset C(\mathbf{R}) \mid X$, and therefore uc $V \subset C(\mathbf{R}) \mid X$ by $1.5(1)$. Thus $h \in($ us $V \cap$ ls $V$ ) - uc $V$.

Our main result on strict insertion is as follows:

3.7. THEOREM. Let $V$ be a uniformly closed vector sublattice of $F(X)$ and let $f, g \in F(X)$. If either $f, g \in F^{*}(X)$ and $V$ is closed under bounded quotients, or if $V$ is inversion-closed, then the following are equivalent:

(1) There exists $h \in V$ such that $h$ is strictly between $f$ and $g$.

(2) There exist $f^{\prime}, g^{\prime} \in F(X)$ such that every upper (resp. lower) Lebesgue set of $f^{\prime}\left(\right.$ resp. $\left.g^{\prime}\right)$ is a $V$-zero-set, $f \leq f^{\prime} \leq g^{\prime} \leq g$, and $f^{\prime}(x)<g(x)$ and $f(x)<g^{\prime}(x)$ whenever $x \in X$ with $f(x)<g(x)$.

ProOF. We assume first that $f, g \in F^{*}(X)$ and that $V$ is closed under bounded quotients. 
(1) $\Rightarrow(2)$. It suffices to take $f^{\prime}=h=g^{\prime}$.

$(2) \Rightarrow(1)$. By 3.4 and the hypotheses on the Lebesgue sets of $f^{\prime}$ and $g^{\prime}, V$ completely separates the Lebesgue sets of $\left\langle f^{\prime}, g^{\prime}\right\rangle$ and hence also those of $\langle f, g\rangle$. By 1.6, there exists $k \in V$ such that $f \leq k \leq g$. Moreover, by 3.2 there exist, respectively, decreasing and increasing sequences $\left\langle f_{n}: n \in \omega\right\rangle$ and $\left\langle g_{n}: n \in \omega\right\rangle$ of functions (which we may assume are bounded) in $V$ such that $f^{\prime}=\bigwedge_{n \in \omega} f_{n}$ and $g^{\prime}=\bigvee_{n \in \omega} g_{n}$. Then (1) follows from (2) by the argument of the proof of $\left[\mathbf{L}_{2}\right.$, Lemma 1].

The case in which $V$ is inversion-closed now follows from what has just been proved together with 2.3 .

3.8. COROLLARY. Let $V$ be a uniformly closed vector sublattice of $F(X)$, let $f, g \in F(X)$ with $f \leq g$, and assume that either $f, g \in F^{*}(X)$ and $V$ is closed under bounded quotients or that $V$ is inversion-closed. If every upper (resp. lower) Lebesgue set of $f$ (resp. $g$ ) is a $V$-zero-set, then there exists $h \in V$ such that $h$ is strictly between $f$ and $g$.

3.9. REMARKS. (a) The implication (ii) $\Rightarrow$ (i) of $\left[\mathbf{L}_{2}\right.$, Theorem 2] is essentially the special case of 3.8 for which $X$ is a topological space, $f, g \in F^{*}(X)$, and $V=C^{*}(X)$.

(b) Mauldin proves the following in [Ma, Theorem 6]: $(*)$ If $V$ is a vector sublattice of $F(X)$ and if $f \in$ us $V$ and $g \in \operatorname{ls} V$ with $f \leq g$, then there exists $h \in$ us $V \cap \operatorname{ls} V$ with $h$ strictly between $f$ and $g$. We note that $(*)$ immediately yields the special case of 3.8 described in (a) and that 3.8 itself follows from $(*)$, 3.2 , and 3.5 .

\section{REFERENCES}

[Ba] R. Baire, Sur les séries à termes continus et tous de même signe, Bull. Soc. Math. France 32 (1904), 125-128.

[B1] R. L. Blair, Extensions of Lebesgue sets and of real-valued functions, Czechoslovak Math. J. 31 (1981), 63-74.

[Bo] N. Bourbaki, Elements of mathematics: general topology, Part 2, Hermann, Paris and AddisonWesley, Reading, Mass., 1966.

[D] J. Dieudonné, Une généralisation des espaces compacts, J. Math. Pures Appl. 23 (1944), 6576.

[E] R. Engelking, General topology, PWN, Warsaw, 1975; English transl., PWN, Warsaw, 1977.

[GJ] L. Gillman and M. Jerison, Rings of continuous functions, University Series in Higher Math., Van Nostrand, Princeton, 1960.

[Ha] A. W. Hager, Real-valued functions on Alexandroff (zero-set) spaces, Comment. Math. Univ. Carolin. 16 (1975), 755-769.

[H] H. Hahn, Über halbstetige und unstetige Funktionen, Sitzungsber. Akad. Wiss. Wien Abt. IIa 126 (1917), 91-110.

[K, $\mathbf{K}_{1}$ M. Katètov, On real-valued functions in topological spaces, Fund. Math. 38 (1951), 85-91.

$\left[\mathbf{K}_{2}\right] \ldots$, Correction to "On real-valued functions in topological spaces", Fund. Math. 40 (1953), 203 205.

$\left[\mathbf{L}_{1}\right]$ E. P. Lane, Insertion of a continuous function, Topology Proc. 4 (1979), 463- 478.

$\left[\mathbf{L}_{2}\right] \_$, Lebesgue sets and insertion of a continuous function, Proc. Amer. Math. Soc. 87 (1983), 539-542.

[Ma] R. D. Mauldin, On the Baire system generated by a linear lattice of functions, Fund. Math. 68 (1970), 51-59.

[M] S. Mrówka, On some approximation theorems, Nieuw Arch. Wisk. (3) 16 (1968), 94 - 111.

[T] H. Tong, Some characterizations of normal and perfectly normal spaces, Duke Math. J. 19 (1952), 289-292.

Department of Mathematics, Ohio University, Athens, Ohio 45701 